J Acquir Immune Defic Syndr. 2017 May 01; 75(1): 27-34. doi:10.1097/QAI.0000000000001299.

\title{
An expanded HIV testing strategy leveraging the electronic medical record uncovers undiagnosed infection among hospitalized patients
}

\author{
Uriel R. Felsen, MD, MPH, MS ${ }^{1}$, Chinazo O. Cunningham, MD, MS ${ }^{1}$, Moonseong Heo, $\mathrm{PhD}^{2}$, \\ Donna C. Futterman, $\mathbf{M D}^{3}$, Jeffrey M. Weiss, MD' ${ }^{1}$, and Barry S. Zingman, MD1 \\ ${ }^{1}$ Department of Medicine, Albert Einstein College of Medicine and Montefiore Medical Center, \\ Bronx, NY \\ ${ }^{2}$ Department of Epidemiology and Population Health, Albert Einstein College of Medicine, Bronx, \\ NY \\ ${ }^{3}$ Department of Pediatrics, Albert Einstein College of Medicine and Montefiore Medical Center, \\ Bronx, NY
}

\section{Abstract}

Background-Routine HIV testing of hospitalized patients is recommended, but few strategies to expand testing in the hospital setting have been described. We assessed the impact of an electronic medical record (EMR) prompt on HIV testing for hospitalized patients.

Methods-We performed a pre-post study at three hospitals in the Bronx, NY. We compared the proportion of admissions of patients 21-64 years old with an HIV test performed, characteristics of patients tested, and rate of new HIV diagnoses made by screening while an EMR prompt recommending HIV testing was inactive vs. active. The prompt appeared for patients with no prior HIV test or a high-risk diagnosis after their last HIV test.

Results-Among 36,610 admissions while the prompt was inactive, 9.5\% had an HIV test performed. Among 18,943 admissions while the prompt was active, $21.8 \%$ had an HIV test performed. Admission while the prompt was active was associated with increased HIV testing among total admissions (aOR 2.78, 95\% CI 2.62-2.96), those without a prior HIV test (aOR 4.03 95\% CI 3.70-4.40), and those with a prior negative test (aOR 1.52, 95\%CI 1.37-1.68) $(\mathrm{p}<0.0001$ for all). While the prompt was active, testing increased across all patient characteristics. New HIV diagnoses made by screening increased from 8.2/100,000 admissions to 37.0/100,000 admissions while the prompt was inactive and active, respectively (OR 4.51 95\% CI 1.17-17.45, $\mathrm{p}=0.03$ ).

Conclusions-An EMR prompt for hospitalized patients was associated with a large increase in HIV testing, a diversification of patients tested, and an increase in diagnoses made by screening.

CORRESPONDENCE. Uriel Felsen, MD, MPH, MS, Montefiore Medical Center, AIDS Center, 111 E $210^{\text {th }}$ St, Bronx, NY 10467, Phone (718)920-8588, Fax (718)405-0610, ufelsen@ montefiore.org.

Aspects of this work were presented at IDWeek 2017, New Orleans, LA.

CONFLICTS OF INTEREST

No other authors have relevant conflicts of interest to report. 


\section{Keywords}

Expanded HIV testing; Undiagnosed HIV; Electronic Medical Record; Hospital

\section{INTRODUCTION}

Undiagnosed HIV--infection with the virus among people unaware of their status--remains an important individual and public health concern. ${ }^{1}$ In the decade since national guidelines first recommended an expansion of both non-risk-based and risk-based HIV screening in medical settings, ${ }^{2}$ multiple strategies have been employed to implement the recommendations. Tested strategies include revised consent requirements, ${ }^{3}$ adaptations of work-flow, ${ }^{4,5}$ and use of prompts in electronic medical records (EMRs). ${ }^{6,7}$ An advantage of EMR prompts is that the interface itself guides users towards the desired outcome, thereby limiting the need for additional training to alter behavior. ${ }^{8}$

Although recommendations for expanded HIV screening include hospitalized patients, most studies of expanded screening focus on outpatient and Emergency Department (ED) settings. ${ }^{9}$ One reason for the lack of focus on hospitalized patients may be that, by virtue of being hospitalized, patient acuity likely supersedes the performance of routine screening tests. Considering the dearth of literature on expanded HIV screening in the hospital setting, questions remain regarding effective strategies to expand screening in this setting and whether such strategies would meaningfully contribute to HIV prevention efforts.

We sought to determine the impact of an HIV testing strategy enhanced by an EMR prompt for hospitalized patients. Specifically, we assessed the impact of the strategy on the (1) proportion of hospital admissions during which an HIV test was performed, (2) clinical and demographic characteristics of patients tested, and (3) rate of new HIV diagnoses made by screening. We hypothesized that, compared to when the prompt was inactive, the strategy would be associated with increased testing among overall admissions, a diversification of the population tested, and an increased rate of new HIV diagnoses made by screening.

\section{METHODS}

\section{Study design}

We conducted a prospective, quasi-experimental pre-post study comparing HIV testing among hospitalized patients while an EMR prompt reminding providers to offer HIV testing was inactive (standard testing phase) vs. active (enhanced testing phase). The study was conducted between September 1, 2013 and March 31, 2015 and was approved by the Institutional Review Board of Albert Einstein College of Medicine.

\section{Setting}

The study was performed at Montefiore Medical Center (MMC), the largest provider of medical care in the Bronx, NY. MMC includes three adult hospitals and a children's hospital, each with an ED, and more than 50 outpatient sites. All sites share an EMR first implemented in 1997 that includes problem lists, visit history with associated diagnostic 
codes, and laboratory results. The EMR also serves as the portal through which provider place orders for hospitalized patients. There are approximately 80,000 admissions annually to the adult hospitals. The population served is more than $80 \%$ black or Hispanic and approximately one-third live below the poverty line. ${ }^{10}$ The estimated prevalence of HIV in the Bronx is $2 \% .^{11}$

\section{Population}

Patients 21 to 64 years old who were admitted to any of MMC's adult hospitals during the study period were eligible for inclusion. Patients younger than 21 are admitted to the children's hospital and were not included in the study. Patients were excluded if an HIV test was performed during the ED portion of their hospitalization or if they were admitted to an obstetric service because separate protocols govern HIV testing in these settings.

\section{Overview of HIV testing during study period}

During both the standard and enhanced testing phases, patients could be offered HIV testing directly by their providers, by HIV counselors responding to orders placed by providers in the EMR, or by counselors approaching patients without an order. In accordance with MMC policy, all offers were opt-in.

From September 1st, 2013 until May 17, 2014 specimens underwent third generation HIV testing (Siemens Medical Diagnostics) with reactive results confirmed by Western blot. From May 18, 2014 to March 31, 2015 specimens underwent fourth generation testing with the HIV Ag/Ab Combo (Abbott). Reactive results were confirmed by HIV 1/2 Multispot (Bio-Rad) or, for those with nonreactive or indeterminate Multispot results, by qualitative viral load (Aptima). Because the transition from third to fourth generation testing occurred while the EMR prompt was active, the prompt was suspended for re-programming. Therefore, the total study period comprised two periods of standard testing alternating with two periods of enhanced testing.

The number of HIV counselors on staff was constant throughout the study. Counselors worked weekdays from 9 a.m. to 5 p.m. and responded to EMR orders placed outside of these hours on the next business day. All counselors were bilingual in English and Spanish.

\section{HIV testing during standard testing phase}

The standard testing phase comprised two periods, September 1, 2013 to March 11, 2014 and May 18, 2014 to November 18, 2014 (total days = 377). During these periods, there was no reminder for providers to offer HIV testing. Providers could offer testing independently or place an order through the EMR for an HIV counselor to offer testing. When counselors were not responding to orders, they determined which patients to approach by manually reviewing medical records for documentation of prior HIV testing, high-risk sex, sexually transmitted infections, substance use, or viral hepatitis. Efforts were focused on those without a documented HIV test and those at risk after a prior negative test. 


\section{HIV testing during enhanced testing phase}

The enhanced testing phase comprised two periods, March 12, 2014 to May 17, 2014 and November 19, 2014 to March 31, 2015 (total days = 199). During these periods, a prompt recommending the offer of HIV testing appeared to providers placing orders for hospitalized patients meeting either of the following criteria: the patient (1) had no HIV test result in the EMR, or (2) had a high-risk diagnosis subsequent to the last documented negative HIV test. The prompt was accompanied by an order-set allowing providers to (1) request that an HIV counselor offer testing; (2) order an HIV test and document that consent was obtained by the provider; or document that the patient (3) declined HIV testing; (4) self-reported being HIVpositive; (5) was unstable or lacked capacity to consent; or (6) was terminally ill. If no option from the order-set was selected, the prompt reappeared each time a provider placed orders in the patient's EMR.

The logic driving the prompt captured all prior HIV tests performed within MMC. The prompt to re-offer testing was programmed to appear if, after the last negative HIV test, a patient had either a visit to MMC or a problem list entry associated with an International Classification of Disease, 9th revision, Clinical Modification (ICD9-CM) code for a highrisk diagnosis. High-risk diagnoses included sexually transmitted infections, hepatitis B or C infection, substance use, HIV indicator conditions, ${ }^{12}$ or AIDS-related conditions. ${ }^{13}$ The list of ICD-9CM codes conferring high-risk is available as supplemental material. Because it was bound to the logic of the prompt, presence of a high-risk diagnosis after an earlier negative HIV test was not available for admissions occurring during the standard testing phase.

\section{Outcomes}

The primary outcome was the proportion of hospital admissions for patients 21 to 64 years old during which an HIV test was performed. Secondary outcomes included the proportion of admissions with a test performed by clinical and demographic characteristics, and rates of new HIV diagnoses made by screening.

\section{Data sources, variables, and definitions}

Data were extracted from the EMR. The predictor of interest was whether an admission occurred during the standard or enhanced testing phase and was determined by the admission date. Other variables included patient demographics, hospital site, admitting service, length of stay, and HIV status at time of admission.

Based on a published algorithm using EMR data, we classified patients as HIV-positive, HIV-negative, or as having unknown HIV status at time of admission. ${ }^{14}$ Patients were considered HIV-positive if they met the algorithm's laboratory, billing, or problem list criteria, or if self-report of HIV-infection was documented in the EMR. Patients were considered HIV-negative if they did not fulfill criteria as being HIV-positive and there was a negative HIV antibody test preceding the admission. All other patients were considered to have unknown HIV status. 
We manually reviewed medical records of all patients with a positive HIV test performed during the study period. Using a standardized chart-abstraction tool, all positive tests were classified as representing (1) a previously known diagnosis or a new diagnosis, and (2) one of three categories of testing-type: corroborative test, diagnostic test, or screening test. A positive test represented a previously known diagnosis if there was documentation in the medical record that the patient was known to be HIV-positive prior to admission. If this condition was not met, the test represented a new diagnosis. A positive test represented corroborative testing if any provider note written prior to the time that the result was available indicated that the patient's HIV-positive status was already known. A positive test represented diagnostic testing if any provider note written prior to the time that the result was available mentioned HIV as a diagnostic consideration. A positive test represented screening if there was no mention of HIV as a past diagnosis or current diagnostic consideration in any provider note written prior to the time that the result was available.

\section{Statistical Analyses}

The unit of analysis was hospital admission. Descriptive statistics are presented as frequencies and proportions for categorical variables and as medians with interquartile ranges (IQR) for continuous variables.

To test the association between study phase and HIV testing, we created multivariable generalized linear mixed models for binary outcomes. Because readmissions are common, patients could be included multiple times during the study. To account for repeated measures within patients we used an $\operatorname{AR}(1)$ covariance structure. The models were adjusted for all covariates associated with HIV testing at a significance level of $\mathrm{p} \leq 0.2$ on bivariate testing. To adjust for secular trends, we included a variable for study-week. Admission diagnosis was not included in the models because of expected collinearity with admitting service. Because the EMR prompt incorporated HIV status at time of admission, we did not include this status as a separate covariate. In preliminary analyses we did not find a significant difference in the magnitude of association between the two non-contiguous standard testing periods or the two non-contiguous enhanced testing periods with HIV testing. Therefore, in the final model the two standard testing periods and the two enhanced testing periods were considered in aggregate. We applied this model to (1) all admissions, (2) admissions restricted to patients with unknown HIV status, and (3) admissions restricted to HIVnegative patients. P-values $<0.05$ were considered statistically significant.

To test for differences in associations between patient characteristics and HIV testing according to study phase, we created additional generalized linear mixed models. These models were stratified by study phase and, in addition to the covariates described above, included HIV status at time of admission. In comparing results from these stratified models, we considered a change in odds ratio (OR) towards a value of one to represent attenuation of an association between a particular characteristic and HIV testing.

To compare rates of HIV diagnoses, we present rates of observed diagnoses per 100,000 admissions. To test for an association between study phase and new HIV diagnoses made by screening, we used generalized linear mixed models with study phase as the independent variable and a new screening diagnosis as the dependent variable. Due to the rarity of this 
outcome, these models were unadjusted. The same analysis was repeated using diagnoses made by corroborative and diagnostic testing as the dependent variable. Analyses used STATA v12 or SAS 9.3.

\section{RESULTS}

Among 70,835 hospital admissions of patients 21 to 64 years old during the study period, 55,553 (78.4\%) met inclusion criteria (Figure). Among these admissions, 36,610 (65.9\%) and 18,943 (34.1\%) occurred during the standard and enhanced testing phases, respectively. The majority of admissions were of females $(55.1 \%)$ and the median age was 51 years (IQR 40-58). Most admissions were of Hispanic (43.4\%) or black (35.8\%) patients, and a substantial minority (11.8\%) was of patients reporting Spanish as their preferred language. Most admissions were to medicine services (66.8\%) and of patients with public insurance (69.7\%). The median length of stay was 3 days (IQR 2-6). Approximately half (51.1\%) of admissions were of patients with unknown HIV status, while $42.6 \%$ and $6.4 \%$ were of HIVnegative and HIV-positive patients, respectively.

Table 1 characterizes the admissions by study phase. Despite some statistical differences, there were no substantial differences in the characteristics of admissions between study phases except that during the enhanced testing phase a greater proportion of admissions were of HIV-negative patients ( $45.2 \%$ vs $41.2 \%$, p $<0.001)$. While presence of a high-risk diagnosis subsequent to a negative HIV test was not captured during the standard testing phase, it was present in $18.1 \%$ of the HIV-negative admissions during the enhanced testing phase.

Table 2 displays the association between study phase and HIV testing, by HIV status. Compared to the standard testing phase, the enhanced testing phase was associated with an increased likelihood of HIV testing among total admissions (adjusted OR [aOR] 2.78, 95\% CI 2.62-2.96), admissions of patients with unknown HIV status (aOR 4.03; 95\%CI 3.70-4.40), and admissions of HIV-negative patients (aOR 1.52; 95\%CI 1.37-1.68). During the enhanced testing phase, among the 1,546 admissions of HIV-negative patients with a subsequent high-risk diagnosis, HIV testing was performed for 568 (36.7\%) (data not shown).

Table 3 displays the proportion of admissions during each study phase with an HIV test performed, by patient characteristic. During the standard testing phase, those who were more likely to be tested included males (compared to females), younger patients (compared to older patients), Hispanic patients (compared to both black and white patients), patients with public insurance (compared to those with private), and patients admitted to medicine services (compared to all other services except neurology). During the enhanced testing phase, the proportion of admissions with an HIV test performed increased across all characteristics. These increases generally attenuated, and in some cases eliminated, independent associations between patient characteristics and the likelihood of an HIV test being performed. Specifically, both the absolute and relative proportions of admissions with a test performed increased among patients who were female, in older age groups, were black or white, had private insurance, and were admitted to non-medicine services. 
During the standard testing phase, there were 48 positive HIV tests. Among these, 19 (39.6\%) were corroborative, 26 (54.2\%) were diagnostic, and 3 (6.3\%) were screening. Among the screening tests, 2 were for patients with a prior negative HIV test and 1 was for a patient with unknown HIV status. During the enhanced testing period, there were 27 positive HIV tests. Among these, 10 (37.0\%) were corroborative, 10 (37.0\%) were diagnostic, and 7 (25.9\%) were screening. Among the screening tests, 1 was for a patient with a prior negative HIV test and subsequent high risk diagnosis and 6 were for patients with unknown HIV status. Table 4 illustrates that the enhanced testing phase was associated with an increased rate of new HIV diagnoses made by screening compared to the standard testing phase (OR $4.51 ; 95 \%$ CI 1.17-17.45). However, the enhanced testing phase was not associated with a different rate of positive diagnostic or corroborative tests (ORs 0.75 ; 95\%CI $0.36-1.54$, and $1.02 ; 95 \%$ CI $0.47-2.19$, respectively).

\section{DISCUSSION}

Our EMR-enhanced HIV testing strategy was associated with increased HIV testing among hospital admissions overall, among those with no prior HIV test, and among those with a prior negative test compared to a strategy with no automated EMR support. The increased volume of testing was also associated with a diversification of patients who were tested for HIV, including among populations with historically lower rates of testing. Most importantly for HIV prevention, this strategy was associated with a 3.5-fold increase in the rate of new HIV diagnoses made by screening compared to the standard testing strategy. These findings provide important insights for those seeking to expand HIV testing among hospitalized patients.

While EMR-based interventions have been effectively used to increase HIV testing in outpatient and ED settings, ${ }^{6,7}$ to our knowledge, ours is the first published study to demonstrate that the EMR can be successfully leveraged to expand HIV testing among hospitalized patients. Because hospitals increasingly use EMRs for order-entry, ${ }^{15}$ elements of our strategy may be reproducible.

The impact of expanded HIV testing is frequently measured by the volume of tests performed. ${ }^{16,17}$ In addition to increasing the numbers of tests, however, a critical goal of expanded testing is reaching populations with historically lower rates of testing. ${ }^{2}$ Our strategy was associated with a diversification of the patients tested. Our finding of increased testing across all measured patient characteristics, and the attenuation of independent associations between those characteristics and performance of an HIV test, reflects an expansion both of volume and populations reached.

There is debate over whether shortcomings historically attributed to targeted testing (i.e. high rates of undiagnosed HIV) reflect deficiencies inherent to targeting or reflect incomplete implementation of comprehensive targeted strategies. ${ }^{18}$ Several studies from EDs comparing targeted and non-targeted strategies suggest that although non-targeted testing may identify a modestly increased number of HIV diagnoses, it does so at a disproportionate expense of limited resources. ${ }^{19-22}$ Importantly, current HIV screening guidelines recommend a combination of non-targeted (testing for all at least once, regardless 
of risk) and targeted (repeat testing for those with ongoing risk) screening. ${ }^{2,23}$ By focusing on those with no documented HIV test regardless of risk and those with known risk after their last negative test, to our knowledge, our strategy represents the closest approximation to implementing the guidelines that is available in the literature. Our finding of increased rates of new HIV diagnoses made by screening suggests that this "middle-ground" strategy may represent a reasonable balance between the strengths and weaknesses of targeted and non-targeted screening.

Notably, we found an increase in new HIV diagnoses made by screening despite multiple large-scale HIV testing initiatives affecting the Bronx. ${ }^{24-26}$ These initiatives are linked to higher rates of HIV-testing among Bronx residents compared to most of the U.S. ${ }^{27}$ Despite the success of these efforts, our findings suggest that in this high-prevalence region, undiagnosed HIV remains a problem even among a population accessing healthcare. Because not all patients in the study were tested, however, it is likely that additional HIV diagnoses were missed. These observations support efforts to further expand HIV testing both within and outside healthcare settings.

An additional strength of our study is the classification of all positive HIV tests as representing screening, diagnostic, or corroborative testing. While most studies comparing the association of different testing strategies with identification of new HIV diagnoses report the number of diagnoses attributable to each strategy, they do not report whether these diagnoses would have likely been made outside the context of expanded testing. This may be because most of these studies took place in EDs where diagnostic HIV testing is unlikely to be as common as it is in inpatient settings. For hospitalized patients, diagnostic testing is the standard of care for patients presenting with signs or symptoms suggestive of HIV infection. Reviewing the medical records of patients with a positive HIV test during hospitalization allowed us to rigorously ascertain the value added by screening to the identification of new HIV diagnoses. While all of the positive screening tests represented new diagnoses, because some HIV-positive patients presenting with HIV-related signs or symptoms had not disclosed their status prior to the HIV test being sent, a small number of the positive diagnostic tests did not represent new diagnoses.

Our study has limitations. First, because our intervention was integrated into the EMR, we were unable to use a randomized design. Additionally, the transition from third to fourth generation testing and the resulting suspension of the intervention were not anticipated. However, we adjusted our analyses for differences in the study populations and for secular trends. Furthermore, both issues reflect the real-world context of our intervention. That our results were observed outside of a closely controlled research setting suggests generalizability to other busy, inpatient settings. Second, our data only includes HIV tests performed within our healthcare system. Because there is no comprehensive patient-level registry of HIV testing we cannot know whether the patients included in our study were ever tested elsewhere. Therefore, our observation that approximately half of the patients were previously tested represents a minimum estimate. Third, only ICD-9CM codes were used to identify high-risk diagnoses triggering a prompt to re-offer testing. Therefore, patients with high-risk behaviors not captured by billing codes such men who have sex with men or those with HIV-positive partners may not have been re-offered testing. Fourth, our strategy 
partially relied on HIV counselors funded through a combination of internal and external mechanisms. Therefore, our results may not be generalizable to settings where counselors are not available. Finally, because our study was performed in a region of high HIV prevalence, our finding of increased new HIV diagnoses is unlikely to be generalizable to regions with different HIV epidemiology.

Our EMR-enhanced HIV testing strategy for hospitalized patients was associated with a large increase in the performance of HIV testing, a diversification of the patients tested, and an increase in the rate of new HIV diagnoses identified by screening compared to a strategy without automated EMR support. Leveraging the EMR to support expanded HIV testing strategies for hospitalized patients can impact key HIV prevention outcomes.

\section{Supplementary Material}

Refer to Web version on PubMed Central for supplementary material.

\section{Acknowledgments}

The authors gratefully acknowledge Montefiore Medical Center's AIDS Center HIV Counseling and Testing team and collaborators at Montefiore Information Technology whose efforts made this work possible.

\section{SOURCES OF FUNDING}

This study was supported in part by the Center for AIDS Research at the Albert Einstein College of Medicine and Montefiore Medical Center (NIH AI-51519); NIH/NCATS UL1TR001073; NIH R25DA023021; NIH K24DA036955; K23MH106386; HRSA U10HA29291; Gilead FOCUS Program for Scaling up HIV Testing; and by the New York City Department of Health and Mental Hygiene HIV though a contract with Public Health solutions (13-SLC-165). Its contents are solely the responsibility of the authors and do not necessarily represent the official views of the funders. BSZ has received research grants from Cepheid to evaluate HIV viral load assays, as well as from Gilead to study antiretroviral agents. DCF has received unrestricted educational and clinical trial grants from Gilead. However, none of this funding is related to the work presented in this manuscript.

\section{REFERENCES}

1. Centers for Disease Control and Prevention (CDC). Monitoring selected national HIV prevention and care objectives by using HIV surveillance data-United States and 6 dependent areas-2012. HIV Surveillance Supplemental Report. 2014; 19(3)

2. Branson BM, Handsfield HH, Lampe MA, et al. Revised recommendations for HIV testing of adults, adolescents, and pregnant women in health-care settings. MMWR. Recommendations and reports : Morbidity and mortality weekly report. Recommendations and reports / Centers for Disease Control. 2006; 55(RR-14):1-17. quiz CE11-14.

3. Zetola NM, Grijalva CG, Gertler S, et al. Simplifying consent for HIV testing is associated with an increase in HIV testing and case detection in highest risk groups, San Francisco January 2003-June 2007. PLoS One. 2008; 3(7):e2591. [PubMed: 18596933]

4. Signer D, Peterson S, Hsieh YH, et al. Scaling Up HIV Testing in an Academic Emergency Department: An Integrated Testing Model with Rapid Fourth-Generation and Point-of-Care Testing. Public Health Rep. 2016; 131(Suppl 1):82-89. [PubMed: 26862233]

5. Futterman D, Stafford S, Meissner P, et al. Ten Sites, 10 Years, 10 Lessons: Scale-up of Routine HIV Testing at Community Health Centers in the Bronx, New York. Public Health Rep. 2016; 131(Suppl 1):53-62. [PubMed: 26862230]

6. Goetz MB, Hoang T, Bowman C, et al. A system-wide intervention to improve HIV testing in the Veterans Health Administration. J Gen Intern Med. 2008; 23(8):1200-1207. [PubMed: 18452045]

7. Lin X, Dietz PM, Rodriguez V, et al. Routine HIV screening in two health-care settings--New York City and New Orleans, 2011-2013. MMWR Morb Mortal Wkly Rep. 2014; 63(25):537-541. 
8. Kawamoto K, Houlihan CA, Balas EA, Lobach DF. Improving clinical practice using clinical decision support systems: a systematic review of trials to identify features critical to success. BMJ. 2005; 330(7494):765. [PubMed: 15767266]

9. Sullivan PS, Lyons MS, Czarnogorski M, Branson BM. Routine Screening for HIV Infection in Medical Care Settings: A Decade of Progress and Next Opportunities. Public Health Rep. 2016; 131(Suppl 1):1-4.

10. United States Census Bureau. [Accessed March 1, 2016] State and County Quickfacts: Bronx County, New York. 2015. http://quickfacts.census.gov/qfd/states/36/36005.html

11. New York City Department of Health and Mental Hygiene. [Accessed February 12, 2016] New York City HIV/AIDS Annual Surveillance Statistics. http://www.nyc.gov/html/doh/html/ah/ hivtables.shtml

12. HIV in Europe. HIV Indicator Conditions: Guidance for implementing HIV testing in Adults in Health Care Settings. 2012 [Accessed April 4, 2016] http://hiveurope.eu/Portals/0/ Guidance.pdf.pdf.

13. Centers for Disease Control and Prevention (CDC). [Accessed April 5, 2016] 1993 Revised Classification System for HIV Infection and Expanded Surveillance Case Definition for AIDS Among Adolescents and Adults. 1993. http://www.cdc.gov/mmwr/preview/mmwrhtml/ 00018871.htm

14. Felsen UR, Bellin EY, Cunningham CO, Zingman BS. Development of an electronic medical record-based algorithm to identify patients with unknown HIV status. AIDS Care. 2014; 26(10): 1318-1325. [PubMed: 24779521]

15. Adler-Milstein J, DesRoches CM, Kralovec P, et al. Electronic Health Record Adoption In US Hospitals: Progress Continues, But Challenges Persist. Health affairs. 2015; 34(12):2174-2180. [PubMed: 26561387]

16. Galbraith JW, Willig JH, Rodgers JB, et al. Evolution and Escalation of an Emergency Department Routine, Opt-out HIV Screening and Linkage-to-Care Program. Public Health Rep. 2016; 131(Suppl 1):96-106. [PubMed: 26862235]

17. Nyaku AN, Williams LM, Galvin SR. Comparison of HIV Testing Uptake in an Urban Academic Emergency Department Using Different Testing Assays and Support Systems. AIDS Patient Care STDS. 2016; 30(4):166-169. [PubMed: 26982908]

18. Haukoos JS, Hopkins E, Bucossi MM. Routine opt-out HIV screening: more evidence in support of alternative approaches? Sex Transm Dis. 2014; 41(6):403-406. [PubMed: 24825339]

19. Haukoos JS, Hopkins E, Conroy AA, et al. Routine opt-out rapid HIV screening and detection of HIV infection in emergency department patients. JAMA. 2010; 304(3):284-292. [PubMed: 20639562]

20. Lyons MS, Lindsell CJ, Ruffner AH, et al. Randomized comparison of universal and targeted HIV screening in the emergency department. J Acquir Immune Defic Syndr. 2013; 64(3):315-323. [PubMed: 23846569]

21. d'Almeida KW, Kierzek G, de Truchis P, et al. Modest public health impact of nontargeted human immunodeficiency virus screening in 29 emergency departments. Arch Intern Med. 2012; 172(1): 12-20. [PubMed: 22025095]

22. Haukoos JS, Hopkins E, Bender B, et al. Comparison of enhanced targeted rapid HIV screening using the Denver HIV risk score to nontargeted rapid HIV screening in the emergency department. Ann Emerg Med. 2013; 61(3):353-361. [PubMed: 23290527]

23. Moyer VA. U.S. Preventive Services Task Force. Screening for HIV: U.S. Preventive Services Task Force Recommendation Statement. Ann Intern Med. 2013; 159(1):51-60. [PubMed: 23698354]

24. [Accessed November 1, 2015] New York City Department of Health and Mental Hygiene. The Bronx Knows HIV Testing Initiative: Final Report. 2012. http://www.nyc.gov/html/doh/ downloads/pdf/ah/bronx-knows-summary-report.pdf

25. Donnell DJ, Hall HI, Gamble T, et al. Use of HIV case surveillance system to design and evaluate site-randomized interventions in an HIV prevention study: HPTN 065. The open AIDS journal. 2012; 6:122-130. [PubMed: 23049660] 
26. State of New York. [Accessed April 5, 2016] Legislation and Memo of Public Health Law 2010 Amendment. http://www.health.ny.gov/diseases/aids/providers/testing/law/docs/ 2010_legislation_and_memo.pdf

27. Myers JE, Braunstein SL, Shepard CW, et al. Assessing the impact of a community-wide HIV testing scale-up initiative in a major urban epidemic. J Acquir Immune Defic Syndr. 2012; 61(1): 23-31. [PubMed: 22739134] 


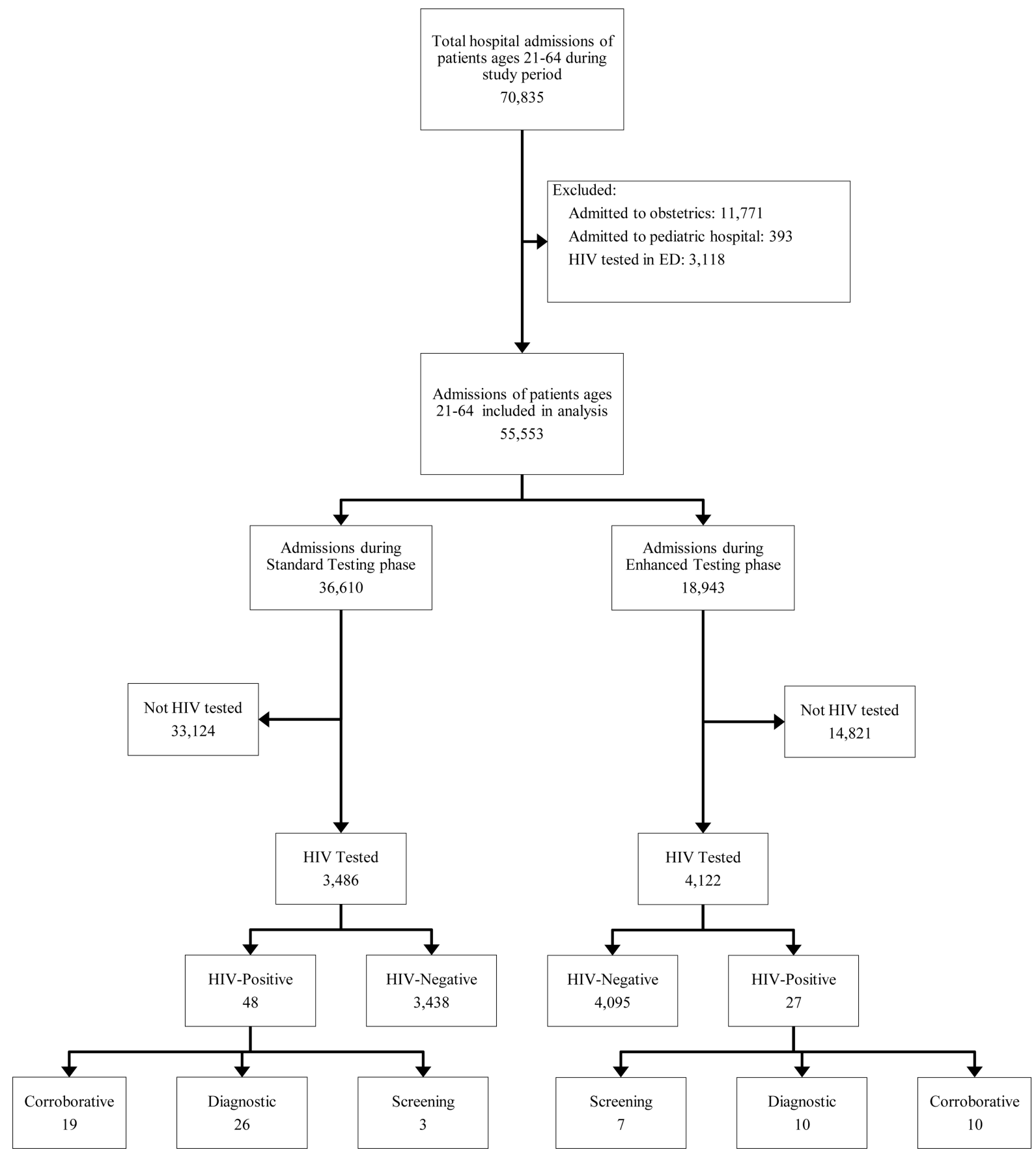

Figure 1.

All hospital admissions of patients ages 21-64 during the study period were considered for inclusion in the analysis. The standard testing phase comprised the periods spanning September 1, 2013 to March 11, 2014 and May 18 2014, to November 18, 2014 (total days= 377). The enhanced testing phase comprised the periods spanning March 12, 2014 to May 17, 2014 and November, 19, 2014 to March 31, 2015 (total days =199). 


\section{Table 1}

Characteristics of hospital admissions by study phase

\begin{tabular}{|c|c|c|c|}
\hline & $\begin{array}{c}\text { Standard Testing } \\
\text { (377 Days) }\end{array}$ & $\begin{array}{c}\text { Enhanced Testing } \\
\text { (199 Days) }\end{array}$ & $\mathbf{p}^{*}$ \\
\hline Characteristic & $\mathbf{N}(\%)$ & $\mathbf{N}(\%)$ & \\
\hline Total & $36,610(100)$ & $18,943(100)$ & \\
\hline Sex & & & 0.47 \\
\hline Female & $20,154(55.1)$ & $10,489(55.4)$ & \\
\hline Age (median years, IQR) & $51(40-58)$ & $51(41-58)$ & $<0.001$ \\
\hline Age (category) & & & $<0.001$ \\
\hline $21-29$ & $3,696(10.1)$ & $1,845(9.7)$ & \\
\hline $30-39$ & $4,960(13.6)$ & $2,603(13.7)$ & \\
\hline $40-49$ & $8,036(22.0)$ & $3,972(21.0)$ & \\
\hline $50-59$ & $13,098(35.8)$ & $6,709(35.4)$ & \\
\hline $60-64$ & $6,820(18.6)$ & $3,814(20.1)$ & \\
\hline Race/ethnicity & & & 0.62 \\
\hline Hispanic & $15,865(43.3)$ & $8,231(43.5)$ & \\
\hline Black, non-Hispanic & $13,145(35.9)$ & 6,747 (35.6) & \\
\hline White, non-Hispanic & $3,848(10.5)$ & $1,974(10.4)$ & \\
\hline Asian, non-Hispanic & $690(1.9)$ & $378(2.0)$ & \\
\hline Other $^{\dagger}$ & $1,945(5.3)$ & $991(5.2)$ & \\
\hline Unknown/Missing & $1,117(3.1)$ & $622(3.3)$ & \\
\hline Preferred Language & & & 0.66 \\
\hline English & $31,403(85.8)$ & $16,251(85.8)$ & \\
\hline Spanish & $4,313(11.8)$ & $2,260(11.9)$ & \\
\hline Other & $659(1.8)$ & $318(1.7)$ & \\
\hline Unknown/Missing & $235(0.6)$ & $114(0.6)$ & \\
\hline Insurance & & & $<0.001$ \\
\hline Public * & $25,499(69.7)$ & $13,228(69.8)$ & \\
\hline Private & $9,674(26.4)$ & $4,832(25.5)$ & \\
\hline Uninsured & $595(1.6)$ & $222(1.2)$ & \\
\hline Unknown/Missing & $842(2.3)$ & $661(3.5)$ & \\
\hline Hospital Site & & & 0.04 \\
\hline A & $9,670(26.4)$ & $5,141(27.1)$ & \\
\hline B & 6,913 (18.9) & $3,658(19.3)$ & \\
\hline $\mathrm{C}$ & $20,027(54.7)$ & $10,144(53.6)$ & \\
\hline Admission Service & & & 0.71 \\
\hline Medicine & $24,488(66.9)$ & $12,596(66.5)$ & \\
\hline Surgery & $8,325(22.7)$ & $4,407(23.3)$ & \\
\hline Neurology & $1,042(2.9)$ & $522(2.8)$ & \\
\hline Psychiatry & $868(2.4)$ & $453(2.4)$ & \\
\hline Rehab Med & $298(0.8)$ & $141(0.7)$ & \\
\hline
\end{tabular}




\begin{tabular}{lccc}
\hline & $\begin{array}{c}\text { Standard Testing } \\
\text { (377 Days) }\end{array}$ & $\begin{array}{c}\text { Enhanced Testing } \\
\text { (199 Days) }\end{array}$ & $\mathbf{p}^{\ddagger}$ \\
\hline Characteristic & $\mathbf{N}(\%)$ & $\mathbf{N}(\%)$ & \\
\hline Gynecology & $1,589(4.3)$ & $824(4.4)$ & \\
Inpatient length of stay (median days, IQR) & $3(2-6)$ & $3(2-6)$ & $<0.001$ \\
HIV Status at time of admission & & & $<0.001$ \\
HIV-Positive & $2,364(6.5)$ & $1,177(6.2)$ & \\
HIV-Negative & $15,076(41.2)$ & $8,564(45.2)$ & \\
$\begin{array}{l}\text { With subsequent high-risk } \\
\text { diagnosis** }\end{array}$ & -- & $1,546(18.1)$ & \\
Unknown HIV Status & $19,170(52.4)$ & $9,202(48.6)$ & \\
\hline
\end{tabular}

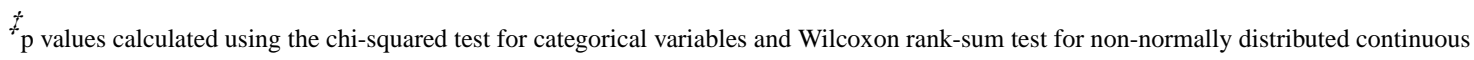
variables

Includes Multiracial, American Indian and Alaskan Native, Native Hawaiian and other Pacific Islander, or other

Medicare or Medicaid

**

Not available during standard phase. In enhanced phase, percentage calculated among those who were HIV-negative. Complete list of high-risk diagnoses available in supplemental material. 
Table 2

Association between study phase and HIV testing by HIV status at time of admission

\begin{tabular}{lcccc}
\hline & Total admitted & Total tested $(\%)$ & OOR (95\% CI) & p \\
\hline Among all admissions & & & & \\
Standard testing & 36,610 & $3,486(9.5)$ & Ref & -- \\
Enhanced testing & 18,943 & $4,122(21.8)$ & $2.78(2.62,2.96)$ & $<0.0001$ \\
\hline $\begin{array}{l}\text { Among admissions of } \\
\text { patients with unknown }\end{array}$ & & & & \\
HIV status & 19,170 & $2,100(11.0)$ & Ref & -- \\
$\begin{array}{l}\text { Standard testing } \\
\text { Enhanced testing }\end{array}$ & 9,202 & $2,979(32.4)$ & $4.03(3.70,4.40)$ & $<0.0001$ \\
\hline $\begin{array}{l}\text { Among admissions of } \\
\text { HIV-negative patients }\end{array}$ & & & & \\
$\begin{array}{l}\text { Standard testing } \\
\text { Enhanced testing }\end{array}$ & 15,076 & $1,376(9.1)$ & $\operatorname{Ref}$ & - \\
\hline
\end{tabular}

* Models adjusted for sex, age, race/ethnicity, preferred language, insurance, hospital site, admissions service, length of stay, and study-week 


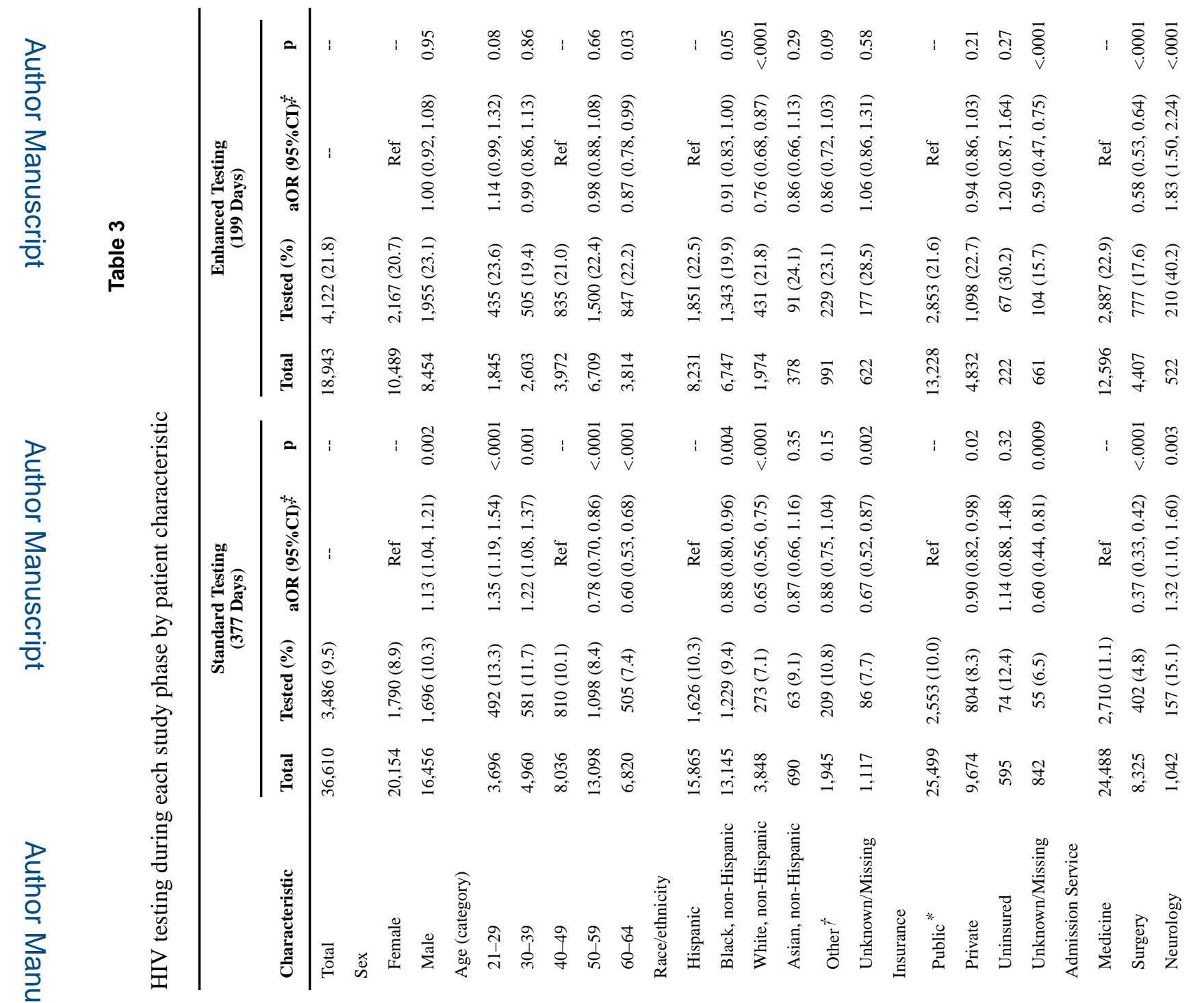


Felsen et al.

Page 17

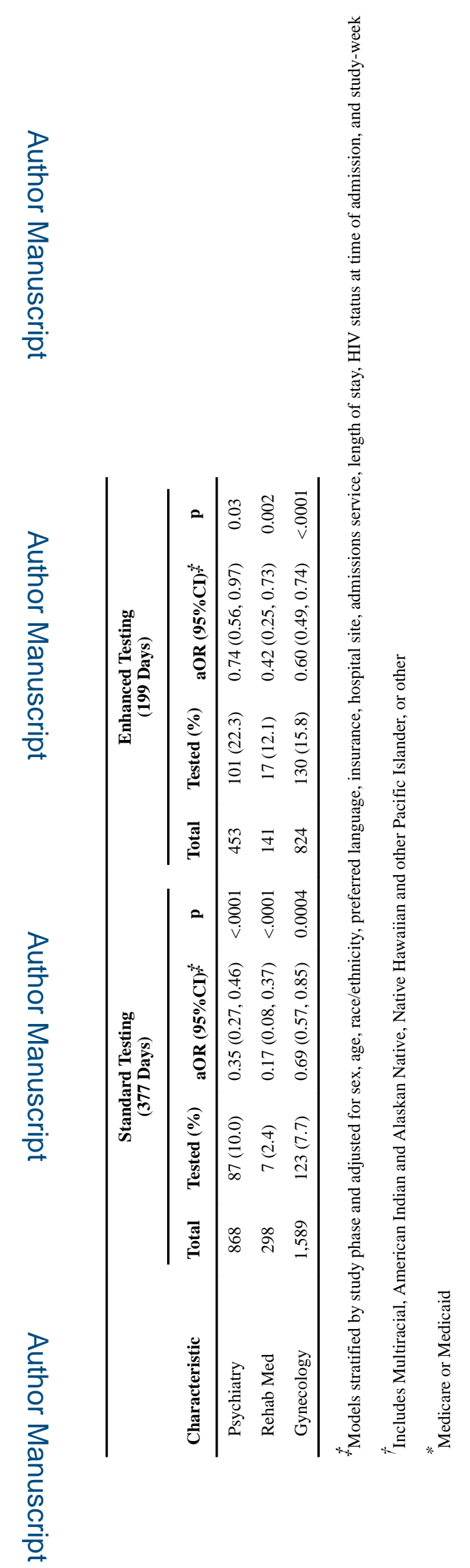

J Acquir Immune Defic Syndr. Author manuscript; available in PMC 2018 May 01. 
